Mena-Mosquera, V. E., \& Andrade C., H. J. (2021). Potencial de reducción de emisiones y captura de carbono en bosques y sistemas agroforestales con cacao en el Pacífico colombiano. Revista de Biología Tropical, 69(4), 12521263. https://doi.org/10.15517/rbt.v69i4.45927

\title{
Potencial de reducción de emisiones y captura de carbono en bosques y sistemas agroforestales con cacao en el Pacífico colombiano
}

Víctor Eleazar Mena-Mosquera1*; (D) https://orcid.org/0000-0001-5163-4220

Hernán J. Andrade C. ${ }^{2}$; (D) https://orcid.org/0000-0002-3398-294X

1. Grupo de Investigación Agroforestería del Trópico Húmedo Chocoano (AGROTROPICO), Programa Ingeniería Agroforestal, Facultad de Ingeniería, Universidad Tecnológica del Chocó, Quibdó, Chocó, Colombia; memovie@gmail.com (Correspondence*)

2. Grupo de Investigación Producción Ecoamigable de Cultivos Tropicales (PROECUT), Departamento de Producción y Sanidad Vegetal, Facultad de Ingeniería Agronómica, Universidad del Tolima, Ibagué, Tolima, Colombia; hjandrade@ut.edu.com

Recibido 22-II-2021. Corregido 29-X-2021. Aceptado 18-XI-2021.

\author{
ABSTRACT \\ Potential for reducing emissions and carbon sequestration in forests and \\ cocoa agroforestry systems in the Colombian Pacific
}

\begin{abstract}
Introduction: Forests and agroforestry systems (AFS) provide ecosystem goods and services for society, such as climate change mitigation.

Objective: The potential for emission reductions and carbon sequestration in forests and cocoa agroforestry systems in the Munguidó river sub-basin in Colombia was estimated.

Methods: Three land use systems were selected (primary forest, secondary forest and AFS with cocoa). Eighteen temporary sampling plots were established, six per system, to measure trees (diameter at breast height $-\mathrm{dbh} \geq 10 \mathrm{~cm}$ ) and cocoa shrubs. Aboveground biomass was quantified with allometric equations and a carbon fraction of 0.5. Carbon fixation in secondary forest and AFS with cocoa was estimated as the ratio of carbon stored to its age. Carbon loss from primary forest was estimated based on deforestation for Chocó and that sub-basin ( 0.6 and $0.3 \%$ /year, respectively). An analysis of variance and LSD Fisher mean comparison was performed to determine differences in carbon storage and carbon sequestration between uses.

Results: Primary Forest stored more carbon than secondary forest and AFS with cocoa (190.1, 22.3 and 5.3 $\mathrm{Mg} / \mathrm{ha}$, respectively). The carbon fixation of secondary forest and AFS with cocoa did not differ (2.23 vs. 1.33 $\mathrm{Mg} / \mathrm{ha} /$ year). In 20 years, the primary forest would present an emission reduction of $1.4-2.6 \mathrm{Tg} \mathrm{CO}_{2}$; and the secondary forest and the PFS with cocoa would present a sequestration of 100.8 and $30.7 \mathrm{Gg} \mathrm{CO}_{2}$, respectively. Conclusion: In the Munguidó river sub-basin, it is possible to establish projects for the reduction of emissions in primary forest and carbon sequestration in secondary forests and AFS with cocoa, which could emit 1.4-2.6 million tons of $\mathrm{CO}_{2}$.
\end{abstract}

Key words: biomass; deforestation; carbon fixation; Munguidó; Chocó.

Los bosques y los sistemas agroforestales (SAF) suministran bienes y servicios ambientales o ecosistémicos valiosos para la humanidad, desde el punto de vista ecológico y económico, los cuales deben ser manejados de forma sostenible para beneficiar a las generaciones futuras (FAO, 2018). Una forma de contribuir a la conservación de los bosques, y de los bienes 
y servicios que éstos proveen, es a través de proyectos que otorguen un pago por el servicio ambiental brindado (Puerta et al., 2018).

Estos ecosistemas están muy relacionados con la problemática del cambio climático global, ya que la vegetación y en especial las leñosas perennes pueden mitigar este problema al capturar el $\mathrm{CO}_{2}$ de la atmósfera y convertirlo en biomasa y carbono que almacenan en forma de madera y vegetación por largos periodos (IPCC, 2007).

De acuerdo con Algensen et al. (2009) la estimación del carbono es relevante porque permite el desarrollo de programas de pago por servicios ambientales (PSA), que a su vez contribuyen a la conservación de los bosques húmedos tropicales $\mathrm{y}$ al desarrollo sostenible de comunidades rurales. En términos de servicios ambientales, por citar un caso específico, se estima que los bosques tropicales contienen hasta $80 \%$ del total del carbono almacenado en toda la vegetación terrestre, siendo determinantes en su ciclo global (Clark, 2007). El Ministerio de Ambiente y Desarrollo Sostenible (MADS, 2017a) señala la importancia de realizar estudios que logren calcular biomasa en los diferentes sistemas forestales y agroforestales, para estimar el carbono fijado o almacenado en estos, valorarlo económicamente y otorgar un PSA.

Los PSA emergen como una alternativa de remuneración por las prácticas sostenibles o de conservación de la biodiversidad en los escenarios rurales (Carbal, 2009). Por esta razón, la utilización de estos instrumentos está creciendo en todo el Mundo y varios países de América Latina están adaptando sus marcos legales e institucionales para favorecer el uso de estos instrumentos. La implementación y fortalecimiento de las áreas protegidas y las regulaciones relativas a la producción maderera, a través de estrategias de gobernanza forestal, son herramientas utilizadas ampliamente para evitar prácticas insostenibles que pueden llevar a la pérdida de bosques (MADS, 2017A; Zanetti et al., 2016). El objetivo del presente estudio fue estimar el potencial de reducción de emisiones y captura de carbono en bosques y sistemas agroforestales con cacao de la subcuenca del río Munguidó, Quibdó, Chocó, Colombia. La fijación de carbono y el potencial de reducción de emisiones determinan los créditos y son elementos clave para el diseño de proyectos de PSA para generar bienestar social, ambiental y económico.

\section{MATERIALES Y MÉTODOS}

Área de estudio: El estudio se realizó en la sub-cuenca del río Munguidó, localizada al margen izquierdo del rio Atrato, al frente de la cabecera municipal de Quibdó ( $5^{\circ} 40^{\prime} 53.72$ " N $\left.\& 76^{\circ} 48^{\prime} 44.45^{\prime \prime} \mathrm{W}\right)$. El área posee una extensión de 61928 ha, un rango de altitud 54 a 200 m y según Holdridge (1996) corresponde a la zona de vida de Bosque pluvial tropical (bp $\mathrm{T})$, la cual presenta una temperatura promedio de $26^{\circ} \mathrm{C}$, una humedad relativa de $94.6 \%$ y una precipitación anual entre 4000 y $10000 \mathrm{~mm}$ (COCOMACIA, 2000; DIAR, 1986; Municipio de Quibdó, 2005).

Selección y establecimiento de unidades de muestreo: Se seleccionaron tres sistemas de uso de la tierra (SUT): bosque primario, bosque secundario y SAF con cacao, considerando que son los más representativos del área y los más importantes para proyectos de carbono en este territorio. El bosque primario con un área de 33255.3 ha es el de mayor cobertura en la subcuenca y presenta una estructura vegetal con una abundancia promedio de 435 individuos/ha, pertenecientes a 50 especies de 46 géneros y 29 familias. Las especies de mayor importancia ecológica son: Chrysophyllum argenteum, Brosimum utile, Protium veneralense, Apeiba tiborbuo y Virola reiidi, con un Índice de Valor de Importancia (IVI) de 26.3, 20.7, 17.7, 17.0, y 15.4, respectivamente. Las familias con mayor representación son: Sapotaceae, Burseraceae, Moraceae, Myristicaceae y Anonaceae (Mena-Mosquera et al., 2020), el bosque secundario con un área de 26 257.5 ha, presenta una estructura vegetal con una abundancia promedio de 435 individuos/ ha, pertenecientes a 50 especies de 46 géneros 


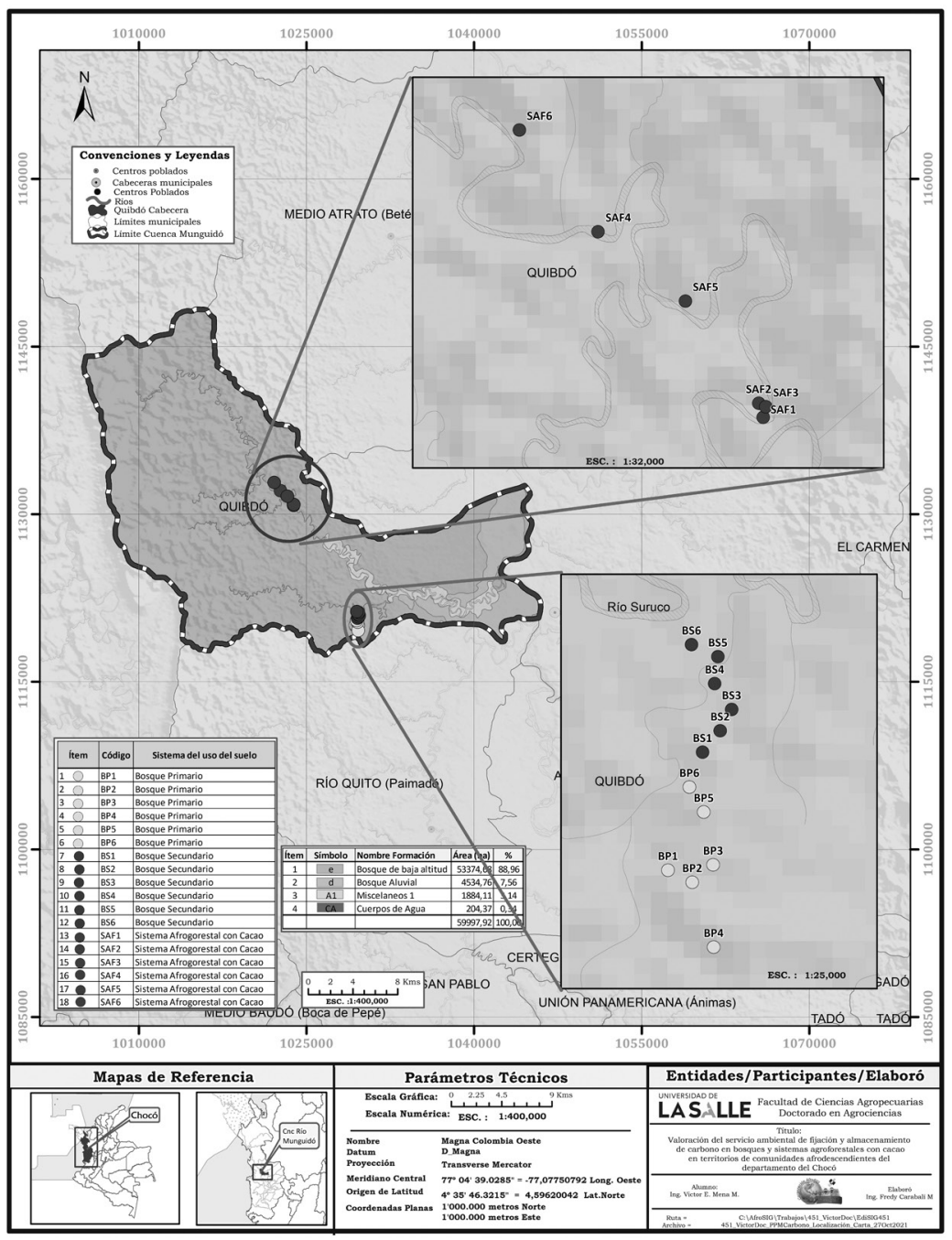

Fig. 1. Localización de unidades de muestreo de biomasa en la subcuenca del río Munguidó, municipio de Quibdó, Chocó, Colombia.

Fig 1. Location of sampling units for biomass in the sub-basin of río Munguidó, municipality of Quibdó, Chocó, Colombia.

y 29 familias. Las especies de mayor importancia ecológica son: Inga sp., Jacaranda capaia, Trichilia poeppigii, Apeiba membranacea y Sacoglotis sp., con un IVI de 48.7; 31.3; 21.0; 17.6, y 15.6, respectivamente. Las familias con mayor representación son: Mimosoideae, Bignoniaceae, Meliaceae, Hypericaceae y Moraceae (Mena-Mosquera et al., 2020) y el SAF con cacao con área 415.2 ha, este cultivo está asociado principalmente a la especie Cedrela odorata, frutales y musáceas, con una abundancia promedio de 500 árboles de cacao/ha y una productividad promedio de $300 \mathrm{~kg} / \mathrm{ha} / \mathrm{año}$ (COCOMACIA, 2000).

En cada SUT se establecieron seis parcelas temporales de muestreo (PTM) de $50 \times 20 \mathrm{~m}$ $\left(1000 \mathrm{~m}^{2}\right)$, para un total de 18 PTM aleatoriamente distribuidas. El establecimiento de las PTM se realizó empleando GPS, brújula y cinta 
métrica, identificando las coordenadas geográficas correspondientes (Fig. 1).

Muestreo y estimación de biomasa aérea: Se midió el diámetro del tronco a la altura del pecho (dap) de todos los fustales (dap $\geq 10 \mathrm{~cm}$ ) y el diámetro del tronco a $30 \mathrm{~cm}$ de altura $\left(\mathrm{d}_{30}\right)$ de todos los arbustos de cacao. Las mediciones se hicieron con forcípula y pie de rey. La biomasa aérea de fustales y arbustos de cacao se estimó con la utilización de ecuaciones alométricas desarrolladas para el ecosistema y especie en estudio.

La biomasa aérea de fustales se estimó utilizando la ecuación desarrollada por Álvarez et al. (2011):

$$
\begin{gathered}
\mathrm{Ba}=\exp (1.595-1.224 * \operatorname{Ln}(\text { dap })+ \\
1.236 *\left(\operatorname{Ln}(\text { dap })^{2}\right)-0.126 *\left(\operatorname{Ln}(\text { dap })^{3}\right)+ \\
0.691 *(\operatorname{Ln}(\mathrm{D})))
\end{gathered}
$$

Donde; $\mathrm{Ba}=$ biomasa aérea de los árboles $(\mathrm{kg}) ; \exp =$ potencia base e; $\mathrm{Ln}=$ logaritmo natural; dap = diámetro del troncoa la altura del pecho $(\mathrm{cm}) ; \mathrm{D}=$ densidad básica de la madera $\left(\mathrm{g} / \mathrm{cm}^{3}\right)$, cuyo valor fue de $0.6 \mathrm{~g} / \mathrm{cm}^{3}$, tal como lo recomienda el IPCC (2006) para América Tropical.

La biomasa aérea de los árboles de sombra en los SAF se estimó utilizando la ecuación desarrollada por Andrade et al. (2008):

$$
\mathrm{Ba}=10^{(1.12+2.62 * \log (\mathrm{dap})+0.03 * \log (\mathrm{h})))}
$$

Donde; $\mathrm{B}$ = biomasa aérea total (kg/árbol); dap $=$ diámetro a la altura del pecho $(\mathrm{cm}) ; \mathrm{h}=$ altura total (m).

La biomasa aérea de arbustos de cacao se estimó utilizando la ecuación desarrollada por Andrade et al. (2008).

$$
\mathrm{B}=10^{\left(-1,625+2,6 * \log \left(\mathrm{d}_{30}\right)\right)}
$$

Donde; $\mathrm{B}=$ biomasa aérea total (kg/árbol); $\mathrm{d}_{30}$ = diámetro del tronco a $30 \mathrm{~cm}$ del suelo $(\mathrm{cm})$.

Estimación del almacenamiento de carbono: El carbono almacenado en la biomasa aérea de los diferentes usos del suelo muestreados, se estimó empleando una fracción de carbono de 0.5 (IPCC, 2006; Rügnitz et al., 2009):

$$
\mathrm{CA}=\mathrm{B} * \mathrm{FC}
$$

Donde; $\mathrm{CA}=$ almacenamiento de carbono en la biomasa aérea $(\mathrm{Mg} / \mathrm{ha}) ; \mathrm{B}=$ biomasa aérea $(\mathrm{Mg} / \mathrm{ha}) ; \mathrm{FC}=$ fracción de carbono.

El almacenamiento total de $\mathrm{CO}_{2}$ para cada SUT fue estimado considerando su promedio del almacenamiento de carbono, su respectiva área y empleando la ecuación propuesta por IPCC (2003).

$$
\mathrm{CO}_{2}=\mathrm{A} * \mathrm{CA} * 3.67
$$

Donde; $\mathrm{CO}_{2}=$ dióxido de carbono $(\mathrm{Mg} / \mathrm{ha}) ; \mathrm{A}$ = área de cada SUT (ha); $\mathrm{CA}=$ almacenamiento de carbono $(\mathrm{Mg} / \mathrm{ha}) ; 3.67$ = factor estequiométrico de conversión a $\mathrm{CO}_{2}$.

Estimación de la fijación de carbono en el bosque secundario y el SAF con cacao: A través de entrevistas con agricultores y madereros del área de estudio, se conoció que el bosque secundario y el SAF con cacao tenían 10 y 4 años en promedio, respectivamente. Se estimaron las tasas de fijación de carbono utilizando la siguiente ecuación:

$$
\mathrm{TFC}=\mathrm{CA} / \mathrm{EA}
$$

Donde: $\mathrm{TFC}=$ tasa de fijación de carbono $(\mathrm{Mg}$ $\mathrm{C} /$ ha/año); $\mathrm{CA}=$ almacenamiento de carbono $(\mathrm{Mg} \mathrm{C} / \mathrm{ha}) ; \mathrm{EA}=$ edad de los árboles en el sistema (años).

Estimación del potencial de reducción de emisiones de carbono en el bosque primario: Se estimó el potencial de reducción de emisiones de $\mathrm{CO}_{2}$ por efecto de la reducción de la deforestación en el bosque primario de la subcuenca del río Munguidó en un periodo de 20 años, tal como lo recomiendan Algensen et al. (2009). La reducción de emisiones se estimó como la diferencia en almacenamiento 
de carbono entre el escenario con proyecto y la situación de referencia (sin proyecto). El escenario con proyecto se definió como aquel en el que se mantiene el carbono en la biomasa al eliminar totalmente la deforestación durante el periodo de simulación. La situación de referencia se concibió como el cambio en el almacenamiento de carbono al aplicar las dos tasas anuales de deforestación de 0.6 y $0.3 \%$ / año, las cuales corresponden a la tasa de pérdida de bosques del departamento del Chocó y la subcuenca del río Munguidó, respectivamente (Cabrera et al., 2011). El almacenamiento de carbono aéreo total en el bosque primario de la subcuenca del río Munguidó se estimó anualmente tomando cada tasa de deforestación, empleando la ecuación sugerida por Segura et al. (2019):

$$
\mathrm{CA}_{\mathrm{i}+1}=\mathrm{CA}_{\mathrm{i}} *\left[\frac{100-\mathrm{t}}{100}\right]
$$

Donde; $\mathrm{CA}_{\mathrm{i}+1}=$ almacenamiento de carbono en el año $i+1$ (Tg); $\mathrm{CA}_{\mathrm{i}}=$ almacenamiento de carbono en el año $i(\mathrm{Tg}) ; t=$ tasa de deforestación $(\% /$ año).

Estimación del potencial de captura de carbono en bosque secundario y en SAF con cacao: La reducción de emisiones y captura de carbono se estimó como la diferencia en almacenamiento de carbono entre el escenario con proyecto y la situación de referencia ( $\sin$ proyecto). En el escenario actual, o sea sin proyecto, para el bosque secundario se asumió una tasa de deforestación de 30 ha/año (600 ha en 20 años), causada por el cambio de uso del suelo, información obtenida a través de conversación con agricultores del área de estudio. El escenario con proyecto se asumió como aquel en donde se elimina totalmente la deforestación del bosque secundario, permitiendo así, que dicha área conserve y capture más carbono en la biomasa aérea durante el periodo de simulación. Se estimó la captura de carbono empleando la siguiente ecuación:

$$
\mathrm{C}=\sum_{\mathrm{t}=1}^{\mathrm{n}} \mathrm{A} *(\mathrm{CA}+\mathrm{t} * \mathrm{TFC})
$$

Donde; $\mathrm{C}=$ captura de carbono $(\mathrm{Gg}) ; \mathrm{A}=$ área del bosque secundario conservado (30 ha/año); $\mathrm{CA}=$ almacenamiento de carbono $(\mathrm{Mg} \mathrm{C} / \mathrm{ha})$; $\mathrm{TFC}=$ tasa de fijación de carbono $(\mathrm{Mg} \mathrm{C} / \mathrm{ha} /$ año); $\mathrm{n}=$ periodo de duración del proyecto (20 años); $\mathrm{t}=$ año estimado.

Para el SAF con cacao, el escenario con proyecto se asumió como aquel en donde se establecen 600 ha de SAF con cacao durante 20 años, es decir, 30 ha/año; permitiendo así, que dicha área capture carbono en la biomasa aérea durante el periodo de simulación. Se estimó la captura de carbono se estimó empleando la siguiente ecuación:

$$
\mathrm{C}=\sum_{\mathrm{t}=1}^{\mathrm{n}} \mathrm{A} *(\mathrm{t} * \mathrm{TFC})
$$

Donde: $\mathrm{C}=$ captura de carbono $(\mathrm{Gg}) ; \mathrm{A}=$ área establecida de SAF (30 ha/año); TFC = tasa de fijación de carbono ( $\mathrm{Mg} \mathrm{C} / \mathrm{ha} / \mathrm{año}) ; \mathrm{n}=$ periodo de duración del proyecto (20 años); $\mathrm{t}=\mathrm{año}$ estimado.

Análisis estadísticos: Se utilizó un diseño completamente al azar con los sistemas de uso del suelo de bosque primario, bosque secundario y SAF con cacao como tratamientos y seis repeticiones, en cada una de las cuales se estableció y midió una PTM. Se establecieron las diferencias del carbono almacenado en la biomasa aérea y la tasa de fijación de carbono entre los sistemas estudiados empleando el análisis de varianza y la prueba de comparación de medias LSD de Fisher, trabajando con una probabilidad del $95 \%$ y un nivel de significancia del $5 \%(\alpha=0.05)$, previo a la comprobación de los supuestos estadísticos. Todos los análisis fueron realizados con el Software InfoStat (Di Rienzo et al., 2017).

\section{RESULTADOS}

Almacenamiento y fijación de carbono en la biomasa aérea: El bosque primario almacenó estadísticamente $(\mathrm{P}<0.05)$ más carbono en la biomasa aérea que el bosque secundario y 


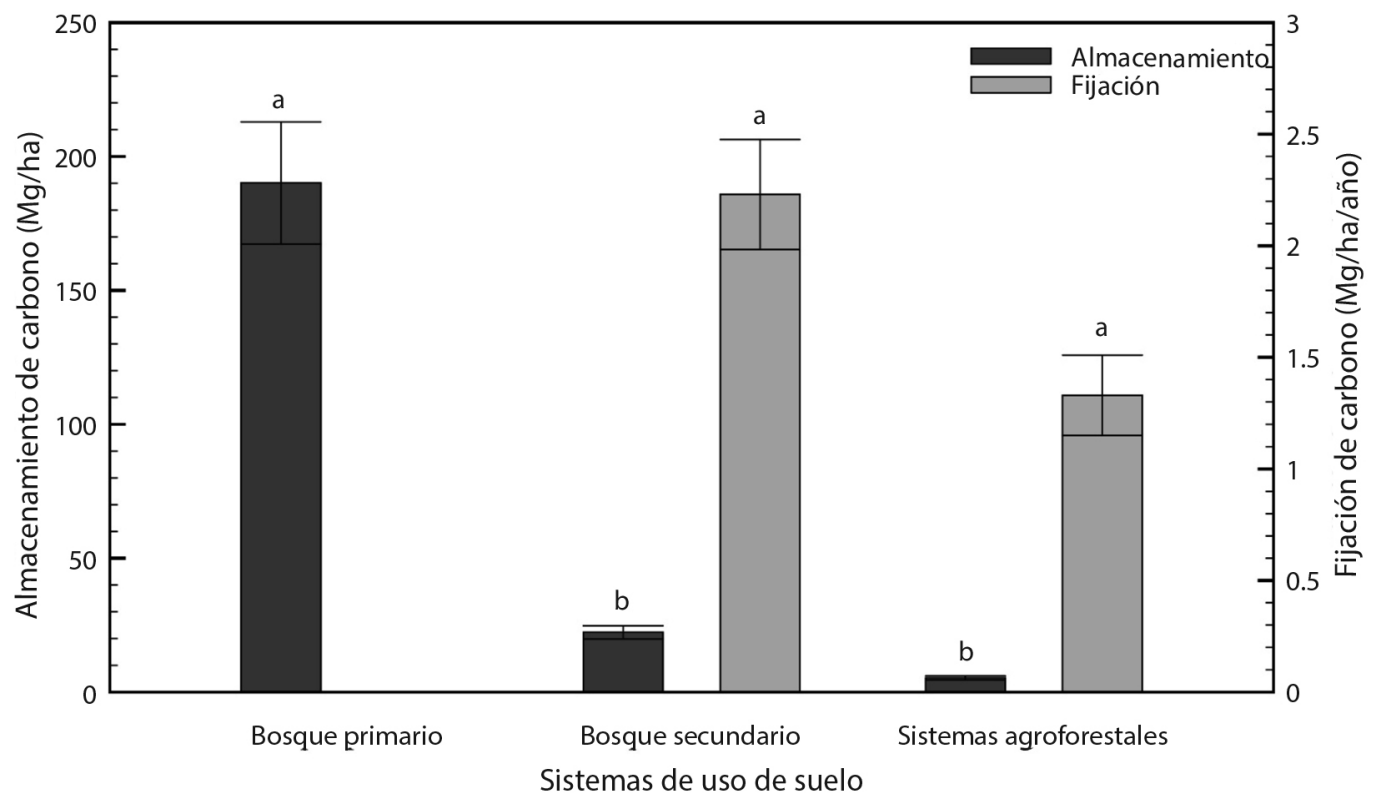

Fig. 2. Almacenamiento y fijación de carbono aéreo de bosques y el SAF con cacao en territorios de comunidades afrodescendientes del río Munguidó, municipio de Quibdó, Chocó, Colombia. Letras diferentes indican que existen diferencias estadísticas entre sistemas de uso del suelo $(\mathrm{P}<0.05)$. Las barras de error corresponden al error estándar.

Fig. 2. Storage and fixation of aboveground carbon in forests and cacao agroforestry systems (AFS) in territories with afrodescendent communities of río Munguidó, municipality of Quibdó, Chocó, Colombia. Different letters indicate statistics differences between land uses $(\mathrm{P}<0.05)$. Error bars correspond to standard error.

el SAF con cacao (190.1 vs 22.3 y $5.3 \mathrm{Mg} / \mathrm{ha}$, respectivamente). Los fustales representan el $99.2 \%$ del carbono almacenado, mientras que los arbustos de cacao solo $0.8 \%$. La subcuenca del rio Munguidó almacenó en total $25.4 \mathrm{Tg}$ de $\mathrm{CO}_{2}$ en donde el bosque primario presentó la mayor contribución $(91.5 \%)$. De otro lado, la fijación de carbono del bosque secundario y el SAF con cacao no presentaron diferencias estadísticas $(\mathrm{P}<0.05)$ entre sí $(2.2$ y $1.3 \mathrm{Mg} /$ ha/año, respectivamente) (Fig. 2).

Potencial de reducción de emisiones y captura de carbono: En un posible proyecto de PSA para la conservación del bosque primario (tipo REDD+), se evitaría una pérdida de entre 1939.5 y 3771.2 ha en 20 años, lo que representaría una reducción de emisiones de carbono de entre 1.4 y $2.6 \mathrm{Tg} \mathrm{CO}_{2}$ durante ese mismo periodo (Fig. 3). En contraste, en un potencial proyecto de PSA con estrategias de captura de carbono, se conservarían 600 ha de bosque secundario, acumulando $100.8 \mathrm{Gg} \mathrm{CO}_{2}$ en 20 años. De igual manera, se implementarían 600 ha de SAF con cacao con una acumulación de $30.7 \mathrm{Gg} \mathrm{CO}_{2}$ durante el mismo periodo (Fig. 4). El bosque secundario y el SAF con cacao presentaron unas tasas de fijación de 2.6 y 1.5 $\mathrm{Gg} \mathrm{CO}_{2}$ /año, respectivamente durante los 20 años de duración de un potencial proyecto.

\section{DISCUSIÓN}

Almacenamiento y fijación de carbono en la biomasa aérea: El bosque pluvial tropical (bp-T) del país posee un área de 206 147 ha y una reserva de carbono en la biomasa aérea de $65.1 \mathrm{Tg} \mathrm{CO}_{2}$. Los bosques primario y secundario de la sub cuenca del río Munguidó representan el $28.8 \%$ del área y el $39.0 \%$ del carbono almacenado en el bosque pluvial tropical de Colombia, lo que demuestra la importancia de este paisaje, lo cual implica que estas áreas deberían tener una alta prioridad 


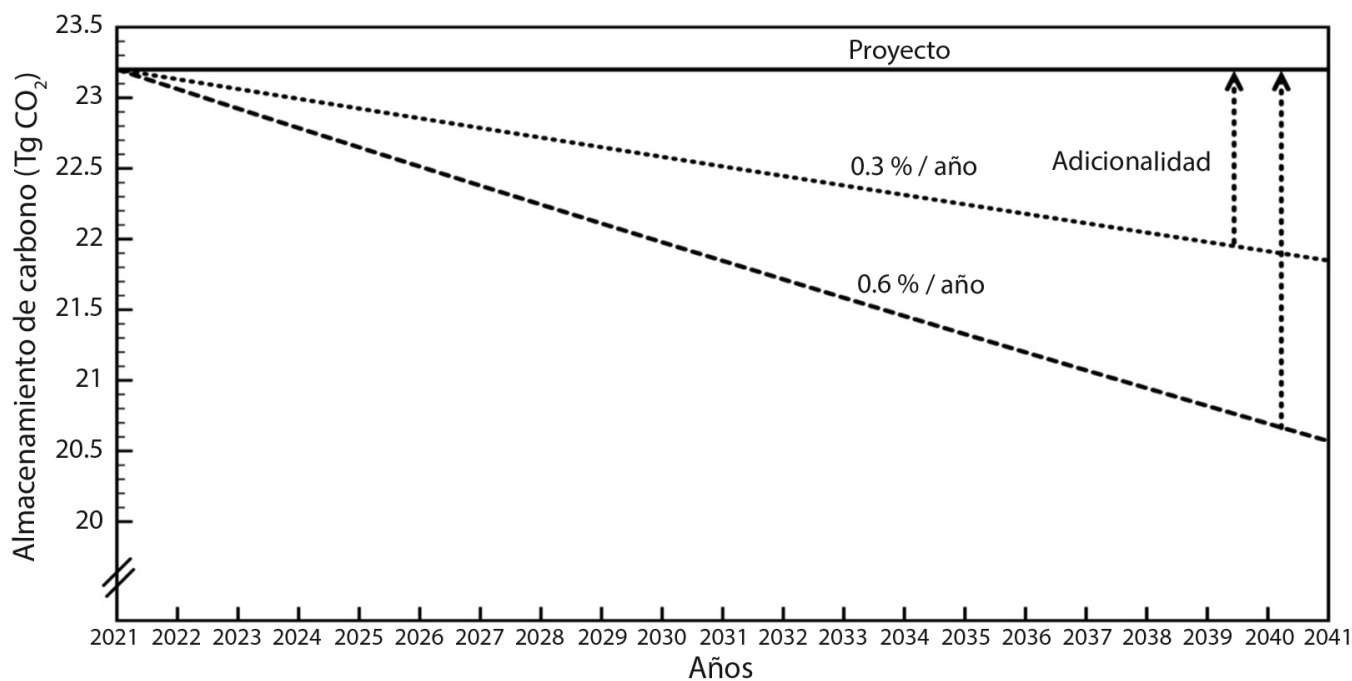

Fig. 3. Potencial de reducción de emisiones de carbono con proyecto de PSA tipo REDD+ en la subcuenca del río Munguidó, municipio de Quibdó, Chocó, Colombia. $1 \mathrm{Tg}=10^{12} \mathrm{~g}$.

Fig. 3. Potential emission reductions of carbon with project of environment services payments (PSE) type REDD + (Reducing Emissions from Deforestation and Degradation) in the sub-basin of río Munguidó, municipality of Quibdó, Chocó, Colombia. $1 \mathrm{Tg}=10^{12} \mathrm{~g}$.

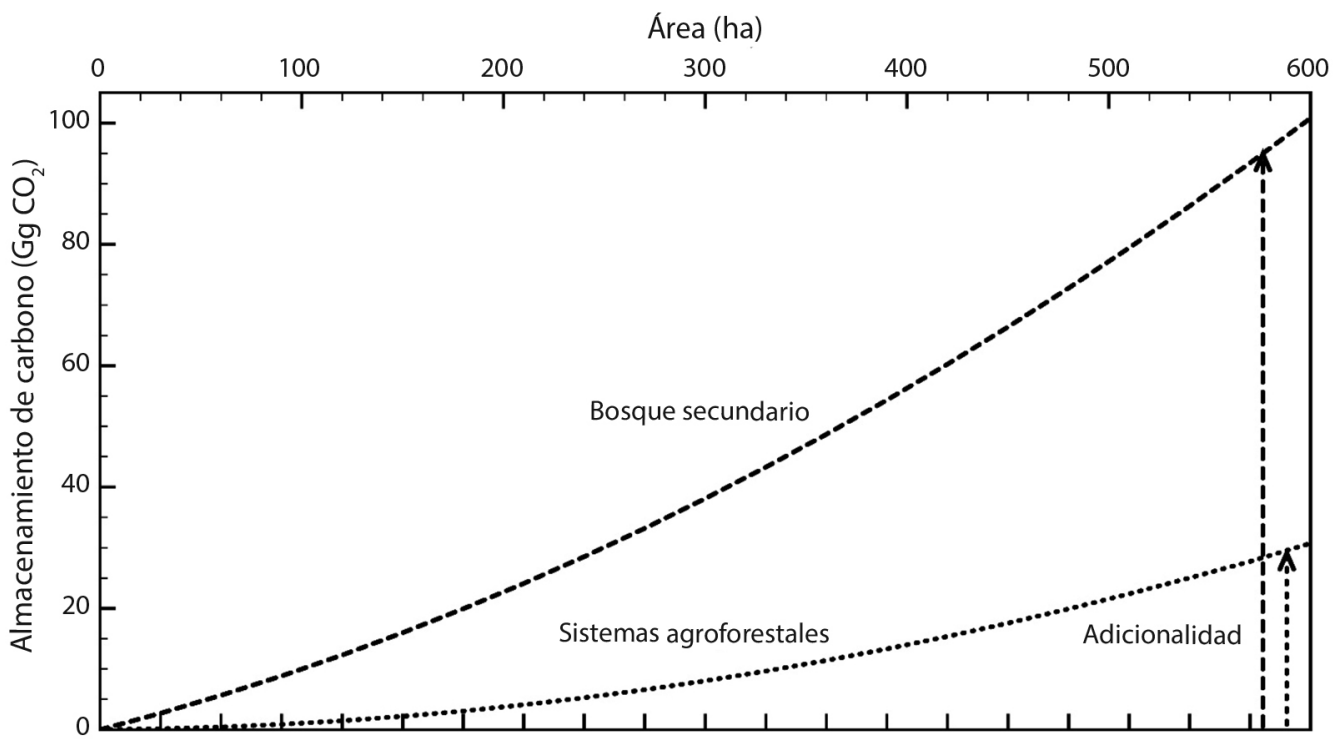

202120222023202420252026202720282029203020312032203320342035203620372038203920402041

Años

Fig. 4. Potencial de captura de carbono en biomasa aérea del bosque secundario y el SAF con cacao con proyecto de PSA en la subcuenca del río Munguidó, municipio de Quibdó, Chocó, Colombia. $1 \mathrm{Gg}=10^{9} \mathrm{~g}$.

Fig. 4. Potential of carbon capture in aboveground biomass of the secondary forests and cacao agroforestry systems (AFS) with project of environment services payments (PSE) in the sub-basin of río Munguidó, municipality of Quibdó, Chocó, Colombia. $1 \mathrm{Gg}=10^{9} \mathrm{~g}$. 
de conservación (Phillips et al., 2011). Las tasas de deforestación presentes en el Pacífico colombiano, representan un alto riesgo para la conservación del bosque pluvial tropical en el país. El carbono almacenado en la biomasa área del bosque primario de la subcuenca del río Munguidó (190.1 Mg C/ha) es similar a los hallazgos de Hurtado et al. (2017) para un bosque primario en la misma zona de vida de Holdridge (219.9 Mg C/ha) y se encuentra en el rango de lo reportado por Chave et al. (2008) para bosques de Colombia (150 a $200 \mathrm{Mg} \mathrm{C} /$ ha). Este ecosistema presenta una tasa de deforestación anual de entre 0.3 y $0.6 \%$ (Cabrera et al., 2011), por lo cual se ha venido reduciendo el área y el almacenamiento total de carbono, produciendo a la vez emisiones de $\mathrm{CO}_{2}$. El almacenamiento de carbono en el bosque secundario (22.3 Mg C/ha) es comparable con los reportes de Torres-Torres et al. (2017) para un bosque húmedo tropical de 12 años en Bahía Solano, Chocó (26.9 Mg C/ha) y es aproximado a las estimaciones de Mena-Mosquera et al. (2011), en la zona de vida bosque muy húmedo tropical en Costa Rica (23.7 Mg C/ha).

Las estimaciones de carbono almacenado en el SAF con cacao a los cuatro años (5.3 $\mathrm{Mg} \mathrm{C} / \mathrm{ha}$ ) fueron inferiores a los hallazgos de Andrade et al. (2013) en cacaotales de 18 y 35 años (28.8 y 33.6 Mg C/ha, respectivamente). Se espera que con la tasa de fijación estimada en este estudio (1.3 Mg C/ha/año), a los 18 años podría alcanzar un almacenamiento de $23.9 \mathrm{Mg}$ $\mathrm{C} /$ ha. Aristizábal y Guerra (2002) afirman que hasta los 15 años hay una alta acumulación de carbono en este sistema de uso de la tierra. La diferencia en el almacenamiento de carbono entre el bosque primario, el bosque secundario y el SAF con cacao, se debe principalmente a la edad entre los ecosistemas, la abundancia de árboles y la presencia de árboles de gran volumen en el bosque primario, como también, al alto grado de intervención antrópica en el bosque secundario y al manejo del SAF con cacao (Marín et al., 2016; Segura et al., 2020).

La fijación de carbono entre el bosque secundario y el SAF con cacao son similares estadísticamente; sin embargo, el bosque secundario posee algo más del doble de la edad del SAF y casi duplica la fijación de carbono. Es probable que el SAF al alcanzar la misma edad, presente similar tasa de fijación que el bosque secundario en este estudio. La fijación de carbono en el bosque secundario $(2.2 \mathrm{Mg} \mathrm{C} /$ ha/año) es superior a la reportada por TorresTorres et al. (2017) para bosques secundarios de 12, 30 y 40 años (1.9 Mg C/ha/año). Estos autores expresan que entre la edad de los bosques secundarios estudiados y la tasa de fijación de carbono hay una relación inversa, ósea, que a medida que aumenta la edad del bosque, disminuye la fijación de carbono. Entre tanto, la fijación de carbono en el SAF con cacao (1.3 Mg C/ha/año), es muy similar al promedio reportado por Andrade et al. (2013) para SAF con cacao de 18 y 35 años (1.1 Mg C/ha/año). La deforestación actual y la dinámica de uso del suelo para actividades agrícolas en el área de estudio representan una amenaza latente frente a la conservación de los bosques y sus servicios ecosistémicos, tal como el almacenamiento y fijación de carbono (Andrade et al., 2017; Segura et al., 2019).

Potencial de reducción de emisiones de carbono en el bosque primario: Los proyectos REDD+ bien concebidos y formulados podrían eventualmente ayudar a detener la deforestación en Colombia y reducir las emisiones de $\mathrm{CO}_{2}$ (Lee \& Pistorius, 2015; Yepes et al., 2011). La subcuenca del río Munguidó almacenó en el bosque primario $25.4 \mathrm{Tg}$ de $\mathrm{CO}_{2}$, si no se establece un proyecto para evitar la tasa de deforestación actual (0.3 y $0.6 \%$ / año, respectivamente), se podrían emitir entre 1.4 y $2.6 \mathrm{Tg}$ de $\mathrm{CO}_{2}$ en 20 años. Estos datos son superiores a los reportados por Segura et al. (2019): $3.2 \mathrm{Tg}$ de $\mathrm{CO}_{2}$ en bosques alto andinos de la Reserva Natural Semillas de Agua (RNSA), de los cuales se podrían emitir entre 119 y $316 \mathrm{Gg} \mathrm{CO}_{2}$ en 20 años a unas tasas de 0.28 y $0.78 \% /$ año, respectivamente. La diferencia en estas proyecciones podría ser explicada por el área, el tipo de bosque y la altitud. Si en el área de estudio no se implementan estrategias de conservación como los proyectos 
REDD+, en el 2100 el bosque primario presentaría una reducción de 23 a $45 \%$ del área y se estarían emitiendo entre 20 y $42 \%$ del carbono almacenado.

Potencial de captura de carbono en el bosque secundario y el SAF con cacao: La regeneración natural en tierras agrícolas brinda la oportunidad de formación de bosques secundarios, los cuales tienen gran potencial para el secuestro y almacenamiento de carbono para la mitigación del cambio climático áreas húmedas del trópico (Martin et al., 2013). Si se logra evitar la tasa de deforestación actual (30 ha/ año) en la subcuenca del río Munguidó, se conservarían 600 ha de bosque secundario en 20 años y se presentaría un potencial de fijación de carbono de $2.6 \mathrm{Gg}$ de $\mathrm{CO}_{2}$. Por otro lado, en ese mismo periodo, el área total del bosque secundario en el área de estudio (26 257 ha) podría capturar y almacenar una cantidad de $\mathrm{CO}_{2}$, equivalente a las emisiones totales producidas por el departamento del Chocó en el año 2012 (4.23 Tg $\mathrm{CO}_{2}$; IDEAM et al., 2016).

Diversos autores han concluido que la fijación de carbono en el SAF es mayor que los monocultivos, ya que la captura de carbono se presenta tanto por los árboles como por los cultivos: (Andrade et al., 2014a; Andrade et al., 2014b; Andrade \& Zapata, 2019; Marín et al. 2016; Orjuela-Chaves et al., 2014). Con el establecimiento de 600 ha de SAF con cacao en la subcuenca del río Munguidó, se presentaría una fijación de carbono de $1.4 \mathrm{Gg}$ de $\mathrm{CO}_{2}$. Estos resultados se convierten en la línea base para la implementación de un proyecto de PSA con este sistema de uso del suelo. Esta estrategia de mitigación del cambio climático comúnmente tiene una alta tasa de adopción, ya que hace parte de la tradición agrícola en el territorio (COCOMACIA, 2000; Municipio de Quibdó, 2005).

En la subcuenca del río Munguidó se posibilita la implementación de proyectos de carbono orientados hacia el manejo de dos estrategias: reducción de emisiones por la conservación del bosque primario y captura de carbono en la biomasa aérea por parte del bosque secundario y SAF can cacao. Este escenario planteado muestra un mayor potencial de emisiones de créditos de carbono por la reducción de emisiones en bosque primario (1.4 a 2.6 millones de toneladas de créditos $\mathrm{CO}_{2}$ ) que por la captura de carbono en bosque secundario y SAF con cacao (131 500 toneladas de créditos $\mathrm{CO}_{2}$ ) (MADS, 2017b; Rontard, 2020; SEMARNAT, 2018). Todas estas estimaciones podrían incrementarse cerca de un $15 \%$ en caso de incluir el componente de biomasa abajo del suelo (Andrade et al., 2021; Cairns et al., 1997); sin embargo, no se incluyó para ser conservadores.

La fijación de carbono y el potencial de reducción de emisiones determinan los créditos y son elementos clave para el diseño de proyectos de PSA para generar bienestar social, ambiental y económico. La potencial emisión y venta de créditos de carbono podría favorecer las estrategias de conservación de bosques y apoyará el establecimiento de SAF con cacao, lo cual redundaría en el mejoramiento del paisaje y el bienestar de las comunidades (ART, 2020; Flores-Aguilar et al., 2018). Los bosques y SAF con cacao en esta área de estudio, además del servicio ambiental de reducción de emisiones, almacenamiento y fijación de carbono, también aportan otros servicios ecosistémicos tal como aquellos derivados de la conservación de la biodiversidad, la regulación hídrica y la belleza escénica (Mena-Mosquera et al., 2020; Torres-Torres et al., 2017).

Declaración de ética: los autores declaran que todos están de acuerdo con esta publicación y que han hecho aportes que justifican su autoría; que no hay conflicto de interés de ningún tipo; y que han cumplido con todos los requisitos y procedimientos éticos y legales pertinentes. Todas las fuentes de financiamiento se detallan plena y claramente en la sección de agradecimientos. El respectivo documento legal firmado se encuentra en los archivos de la revista. 


\section{AGRADECIMIENTOS}

Los autores desean agradecer al Consejo Comunitario Mayor de la Asociación Campesina Integral del Atrato COCOMACIA, Zona 2 Río Munguidó. De la misma forma, agradecemos a la Gobernación del Chocó y la Universidad Tecnológica del Chocó "Diego Luis Córdoba por su apoyo financiero. A los integrantes del Grupo de Investigación Agroforestería del Trópico Húmedo Chocoano (AGROTROPICO) por su aporte en la fase de campo, a Edinson Córdoba Córdoba y David Fernando Pérez Abadía por el apoyo en la construcción de las ecuaciones y a Fredy Carabalí Mosquera por su aporte en la elaboración de los mapas.

\section{RESUMEN}

Introducción: Los bosques y sistemas agroforestales (SAF) suministran bienes y servicios ecosistémicos para la sociedad, tal como la mitigación del cambio climático.

Objetivo: Se estimó el potencial de reducción de emisiones y captura de carbono en bosques y SAF con cacao de la subcuenca del río Munguidó, Colombia.

Métodos: Se seleccionaron tres sistemas de uso del suelo (bosque primario, bosque secundario y SAF con cacao). Se establecieron 18 parcelas temporales de muestreo, seis por sistema, para medir los árboles (diámetro a la altura del pecho -dap $\geq 10 \mathrm{~cm}$ ) y arbustos de cacao. Se cuantificó la biomasa aérea con ecuaciones alométricas y una fracción de carbono de 0.5 . Se estimó la fijación de carbono en el bosque secundario y el SAF con cacao como la razón entre el carbono almacenado y su edad. La pérdida de carbono del bosque primario se estimó con base en la deforestación para Chocó y dicha subcuenca ( 0.6 y $0.3 \%$ /año, respectivamente). Se realizó un análisis de varianza y comparación de medias LSD Fisher para determinar las diferencias en el almacenamiento y la fijación de carbono entre los usos. Resultados: El bosque primario almacenó más carbono que el bosque secundario y el SAF con cacao $(190.1,22.3$ y $5.3 \mathrm{Mg} / \mathrm{ha}$, respectivamente). La fijación de carbono del bosque secundario y el SAF con cacao no difirieron (2.23 vs $1.33 \mathrm{Mg} /$ ha/año). En 20 años, el bosque primario presentaría una reducción de emisiones de 1.4-2.6 $\mathrm{Tg} \mathrm{CO}_{2}$; y el bosque secundario y el SAF con cacao presentarían una captura de 100.8 y $30.7 \mathrm{Gg} \mathrm{CO}_{2}$, respectivamente.

Conclusiones: En la subcuenca del río Munguidó es posible establecer proyectos para la reducción de emisiones en bosque primario y captura de carbono en bosques secundarios y SAF con cacao, con lo cual se podría emitir 1.4-2.6 millones de toneladas de $\mathrm{CO}_{2}$.
Palabras clave: biomasa; deforestación; fijación de carbono; Munguidó; Chocó.

\section{REFERENCIAS}

Algensen, A., Brown, S., Lansel, C., Pesebel, L., Streck, C. H., \& Zarin, D. (2009). Reducción de emisiones de la deforestación y la degradación de bosques (REDD): Reporte de evaluación de opciones. Meridian Institute. Washington, D.C., USA. http://www.redd-oar.org/ links/REDD_OAR_es.pdf

Álvarez, E., Saldarriaga, J. G., Duque, A. J., Cabrera, K. R., Yepes, A. P., Navarrete, D. A., \& Phillips, J. F. (2011). Selección y validación de modelos para la estimación de la biomasa aérea en los bosques naturales de Colombia. Instituto de Hidrología, Meteorología y Estudios Ambientales-IDEAM, Colombia.

Andrade, H. J., Figueroa, J., \& Silva, D. (2013). Almacenamiento de carbono en cacaotales (Theobroma cacao) en Armero-Guayabal. Tolima. Colombia. Scientia Agroalimentaria, 1, 6-10.

Andrade, H. J., Marín, L. M., \& Pachón, D. P. (2014a). Fijación de carbono y porcentaje de sombra en sistemas de producción de café (Coffea arabica L.) en el Líbano, Tolima, Colombia. Bioagro, 26(2) 127-132.

Andrade, H. J., Segura, M. A., Canal, D. S., Feria, M., Alvarado, J. J., Marín, L. M., Pachón, D., \& Gómez, M. J. (2014b). The carbon footprint of coffee productive chains in Tolima, Colombia. In M. Oelberman (Ed.), Sustainable Agroecosystems in Climate Change Mitigation (pp. 53-66). Wageningen Academic Publishers.

Andrade, H. J., Segura, M. A., Canal, D. S., Huertas, A., \& Mosos, C. A. (2017). Composición florística y reservas de carbono en bosques ribereños en paisajes agropecuarios de la zona seca del Tolima, Colombia. Revista de Biología Tropical, 65(4), 1245-1260.

Andrade, H. J., Segura, M. A., Somarriba, E., \& Villalobos, M. (2008). Valoración biofísica y financiera de la fijación de carbono por uso del suelo en fincas cacaoteras indígenas de Talamanca, Costa Rica. Agroforestería en las Américas, 46, 89-96. https://pdfs.semanticscholar.org/e965/60e64cbf25cc67c09d70de45dd16bf2 591d2.pdf

Andrade, H. J., Segura, M. A., \& Feria, M. (2021). Allometric models for estimating belowground biomass of individual coffee bushes growing in monoculture and agroforestry systems. Agroforestry Systems, 95(1), 215-226. https://doi.org/10.1007/ s10457-020-00575-6.

Andrade, H. J., \& Zapata, P. C. (2019). Mitigation of climate change of coffee production systems in Cundinamarca, Colombia. Floresta e Ambiente, 26(3), e20180126. 
Aristizábal, J., \& Guerra, A. (2002). Estimación de la tasa de fijación de carbono en el sistema agroforestal nogal cafetero Cordia alliodora-cacao Theobroma cacao y plátano-Musa paradisíaca (Tesis de grado). Universidad Distrital, Bogotá.

ART (Architecture for REDD+ Transactions). (2020). Arquitectura para las transacciones de REDD+. https://www.artredd.org

Cabrera, E., Vargas, D. M., Galindo, G., García, M. C., Ordoñez, M. F., Vergara, L. K., Pacheco, A. M., Rubiano, J. C., \& Giraldo, P. (2011). Memoria técnica de la cuantificación de la deforestación histórica nacional - escalas gruesa y fina. Instituto de Hidrología, Meteorología, y Estudios Ambientales-IDEAM, Colombia.

Cairns, M., Brown, S., Helmer, E., \& BauMgardner, G. (1997). Root biomass allocation in the world's upland forests. Oecologia, 111, 1-11.

Carbal, H. A. (2009). La valoración económica de bienes y Servicios Ambientales como herramienta estratégica para la conservación y uso sostenible de los ecosistemas: "Caso Ciénaga La Caimanera, Coveñas - Sucre, Colombia”. Colombia. Criterio Libre, 7(10), 71-89.

Chave, J., Olivier, J., Bongers, F., Châtelet, P., Forget, P. M., Van Der Meer, P., Norden, N., Riéra, B., \& Charles-Dominique, P. (2008). Above-ground biomass and productivity in a rain forest of eastern South America. Journal of Tropical Ecology, 24, 355-366.

Clark, D. A. (2007). Detecting tropical forests responses to global climatic and atmospheric change: current challenges and a way forward. Biotropica, 39, 4-19.

COCOMACIA (Consejo Comunitario Mayor del Medio Atrato). (2000). Plan de Manejo Ambiental del Concejo Comunitario Mayor del Medio Atrato: Documento de caracterización. Quibdó. Colombia. COCOMACIA.

Desarrollo Integral Agrícola Rural. (1986). Evaluación de tierras de la región del medio Atrato-Chocó. Quibdó. Colombia. Tomo 2. Codechoco.

Di Rienzo, J. A., Balazarini., M. G., Casanoves, F., Gonzalez, L. A., \& Tablada, E. M. (2017). InfoStat versión 2017. Grupo InfoStat. http://www.infostat.com.ar

FAO (Organización de las Naciones Unidas para la Alimentación y la Agricultura). (2018). El estado de los bosques del Mundo - Las vías forestales hacia el desarrollo sostenible. Roma. Licencia: CC BY-NCSA 3.0 IGO. http://www.fao.org/3/i9535es/i9535es. pdf

Flores-Aguilar, A., Aguilar-Robledo, M., Reyes, H., \& Guzmán-Chávez, M. G. (2018). "Gobernanza ambiental y pagos por servicios ambientales en América Latina". Sociedad y Ambiente, 16, 7-31. doi. org/10.31840/sya.vi22.2106
Holdridge, L. R. (1996). Ecología basada en zonas de vida. Instituto Interamericano de Cooperación para la Agricultura, San José de Costa Rica, IICA.

Hurtado, C. C., Corte, C. H., \& Triana, M. A. (2017). Estimación del Carbono Almacenado en el Bosque Natural en la Cuenca Media-Baja. Municipio de Río Quito, Chocó, Colombia. Ingeniería e Innovación, 9(1), 18-29.

IDEAM, PNUD, MADS, DNP, \& CANCILLERÍA. (2016). Inventario nacional y departamental de Gases Efecto Invernadero - Colombia. Tercera Comunicación Nacional de Cambio Climático. IDEAM, Colombia.

IPCC (Intergovernmental Panel on Climate Change). (2003). Good practice guidance for land use, landuse change and forestry. http://www.ipcc-nggip-iges. or.jp

IPCC (Intergovernmental Panel on Climate Change). (2006). Guidelines for National Greenhouse Gas Inventories. Prepared by the National Greenhouse Gas Inventories Programme, H. S. Eggleston, L. Buendia, K. Miwa, T. Ngara, \& K. Tanabe (Eds.). IGES.

IPCC (Intergovernmental Panel on Climate Change). (2007). Cambio climático: Informe de sintesis. Contribución de los Grupos de trabajo I, II y III al Cuarto Informe de evaluación del Grupo Intergubernamental de Expertos sobre el Cambio Climático. IPCC, Colombia.

Lee, D., \& Pistorius, T. (2015). The impacts of international REDD + finance. The Climate and Land Use Alliance.

Marín, M. P., Andrade, H. J., \& Sandoval, A. P. (2016). Fijación de carbono atmosférico en la biomasa total de sistemas de producción de cacao en el Departamento del Tolima, Colombia. Revista UDCA Actualidad \& Divulgación Cientifica, 19(2), 351-360.

Martin, P. A., Newton, A. C., \& Bullock, J. M. (2013). Carbon pools recover more quickly than plant biodiversity in tropical secondary forests. Proceedings of the Royal Society B: Biological Sciences, 280(1773), 20132236. http://dx.doi.org/10.1098/rspb.2013.2236

Mena-Mosquera, V. E., Andrade, H. J., \& Navarro, C. M. (2011). Biomasa y carbono almacenado en sistemas agroforestales con café y en bosques secundarios en una gradiente altitudinales en Costa Rica. Agroforestería Neotropical, 1(1), 2-20. http://repository.ut.edu. co/handle/001/1278

Mena-Mosquera, V. E., Andrade., H. J., \& Torres-Torres, J. J. (2020). Composición florística, estructura y diversidad del bosque pluvial tropical de la subcuenca del río Munguidó, Quibdó, Chocó, Colombia. Entramado [en línea], 16(1), 204-215. https://doi. org/10.18041/1900-3803/entramado.1.6109 
MADS (Ministerio de Ambiente y Desarrollo Sostenible). (2017a). Decreto 1791 de 1996. Por el cual se establece el pago por Servicios Ambientales y otros incentivos a la conservación. http://es.presidencia. gov.co/normativa/normativa/DECRETO $\% 20870 \% 20$ DEL\%2025\%20DE\%20MAYO\%20DE\%202017.pdf

MADS (Ministerio de Ambiente y Desarrollo Sostenible). (2017b). Decreto 870 del 25 de mayo de 2017: Por el cual se establece el Pago por Servicios Ambientales y otros incentivos a la conservación. https://medioambiente.uexternado.edu.co/ decreto-ley-870-de-2017-por-el-cual-se-establece-elpago-por-servicios-ambientales-y-otros-incentivos-ala-conservacion

Municipio de Quibdó. (2005). Plan de Ordenamiento Territorial - Municipio de Quibdó: Diagnostico de estructura general del territorio. Quibdó. https:// www.quibdo-choco.gov.co/Proyectos/NuestrosPlanes/Plan de Ordenamiento Territorial - Municipio de Quibdó.pdf

Orjuela-Chaves, J. O., Andrade, H. J., \& Vargas-Valenzuela, Y. (2014). Potential of carbon storage of rubber (Hevea brasiliensis Müll. Arg.) plantations in monoculture and agroforestry systems in the Colombian Amazon. Tropical and Subtropical Agroecosystems, 17, 231-240.

Phillips J. F., Duque A. J., Yepes A. P., Cabrera K. R., García, M. C., Navarrete, D. A., Álvarez, E., \& Cárdenas, D. (2011). Estimación de las reservas actuales (2010) de carbono almacenadas en la biomasa aérea en bosques naturales de Colombia. Estratificación, alometría y métodos analíticos. IDEAM, Colombia. http://documentacion.ideam.gov.co/openbiblio/bvirtual/022100/.pdf

Puerta, J. F., Yánez, M. M., \& Medina, R. (2018). Los sistemas de pagos por servicios ambientales de los bosques, una necesidad para el desarrollo sostenible. Universidad y Sociedad, 10(2), 209-216. http://rus. ucf.edu.cu/index.php/rus
Rontard, B., Reyes, H., \& Aguilar, M. (2020). Pagos por captura de carbono en el mercado voluntario en México: diversidad y complejidad de su aplicación en Chiapas y Oaxaca. Sociedad y Ambiente, 22, 212-236. http://dx.doi.org/10.31840/sya.vi22.2106

Rügnitz, M. T., Chacón, M. L., \& Porro, R. (2009). Guía para la determinación de carbono en pequeñas propiedades rurales 1. Lima, Perú. Centro Mundial Agroforestal (ICRAF) / Consorcio Iniciativa Amazónica (IA).

SEMARNAT (Secretaría del Medio Ambiente y Recursos Naturales). (2018). Acuerdo por el que se establecen las bases preliminares del programa de prueba del sistema de comercio de emisiones. http://www.cofemersimir.gob.mx/expedientes/22405

Segura, M. A., Andrade, H. J., \& Mojica, C. A. (2019). Estructura, composición florística y almacenamiento de carbono en bosques nativos del páramo de Anaime, Tolima, Colombia. Ciencia Forestal, 29(1), 57-168. https://doi.org/10.5902/1980509826551

Segura, M. A., Andrade, H. J., \& Sierra, E. (2020). Diversidad florística y captura de carbono en robledales y pasturas con árboles en Santa Isabel, Tolima, Colombia. Revista de Biología Tropical, 68(2), 383-393.

Torres-Torres, J. J., Mena-Mosquera, V. E., \& ÁlvarezDávila, E. (2017). Carbono aéreo almacenado en tres bosques del Jardín Botánico del Pacífico, Chocó, Colombia. Entramado, 13(1), 200-209.

Yepes, A., Navarrete, D. A., Phillips, J. F., Duque, A. J., Cabrera, E., Galindo, G., Vargas, D., García, M. C., \& Ordoñez, M. F. (2011). Estimación de las emisiones de dióxido de carbono generadas por deforestación durante el periodo 2005-2010. IDEAM, Colombia.

Zanetti, E. A., Gómez, J. J., Mostacedo, J., \& Reyes, O. (2016). Cambio climático y politicas públicas forestales en América Latina Una visión preliminar. CEPAL. https://www.cepal.org/es/publicaciones/40922-cambio-climatico-politicas-publicas-forestales-americalatina-vision-preliminar 